\title{
Comprehensive analysis of the prognostic value of the chemokine-like factor-like MARVEL transmembrane domain-containing family in gastric cancer
}

\author{
Zhikun Liang ${ }^{\#}$, Jingwen Xie ${ }^{\#}$, Lihong Huang, Yaoyao Huang, Yuwen Zhang, Ruanxin Ma, Zhuoling Zheng, \\ Qinbo Wang, Xiaoyan $\mathrm{Li}^{\wedge}$
}

Department of Pharmacy, the Sixth Affiliated Hospital, Sun Yat-Sen University, Guangzhou, China

Contributions: (I) Conception and design: Z Liang, J Xie; (II) Administrative support: X Li; (III) Provision of study materials or patients: L Huang,

Y Huang, Y Zhang; (IV) Collection and assembly of data: R Ma, Z Zheng, Q Wang; (V) Data analysis and interpretation: Z Liang, J Xie; (VI) Manuscript writing: All authors; (VII) Final approval of manuscript: All authors.

\#These authors contributed equally to this work.

Correspondence to: Dr. Xiaoyan Li. Department of Pharmacy, the Sixth Affiliated Hospital of Sun Yat-Sen University, Sun Yat-Sen University, 26 Erheng Road of Yuan Village, Tianhe District, Guangzhou 510655, China. Email: lixyan5@mail.sysu.edu.cn.

Background: The chemokine-like factor (CKLF)-like MARVEL transmembrane domain-containing (CMTM) family refers to a family of transcriptional repressor genes. CMTMs are closely associated with the epigenetic regulatory mechanisms and development of multiple malignancies, including gastric cancer. However, their specific biological functions and prognostic values in gastric cancer have yet to be elucidated. Methods: Tumor sample datasets were retrieved and analyzed using databases including Oncomine, STRING, GEPIA2, cBioportal, and Kaplan-Meier plotter. To investigate the prognostic role of CMTMs in gastric cancer, we applied unsupervised hierarchical clustering analysis of CMTM gene expression patterns.

Results: While the mRNA levels of CMTM1/3/6/7/8 were upregulated in gastric cancer, CMTM2/4/5 showed no statistically significant difference at the mRNA level in patients with gastric cancer. Moreover, the mRNA expressions of different CMTMs exhibited strong correlations with various clinical parameters of patients with gastric cancer, including tumor stage, metastatic lymph node status, $H$. pylori status, and tumor grade. Also, the results suggested that elevated levels of CMTM $3 / 5$ mRNA had a significant association $(\mathrm{P}<0.05)$ with poor overall survival, progression-free survival, and post-progression survival. Conversely, elevated expression of CMTM2/4/6 mRNA had a significant association with better overall survival, progression-free survival, and post-progression survival. Unsupervised hierarchical clustering analysis successfully identified 2 major clusters of patients as follows: signature \#1: CMTM4/6/8 and signature \#2: CMTM1/2/3/5/7. Signature \#2 was closely correlated with poorer overall survival, which indicated that the expression pattern of the CMTM family could be a novel prognostic factor for patients with gastric cancer.

Conclusions: These results suggest that the expression levels of CMTM genes possibly have prognostic value as a biomarker of gastric cancer.

Keywords: Gastric cancer; CMTM family genes; prognosis; biomarkers; unsupervised hierarchical clustering

Submitted Jan 07, 2021. Accepted for publication Apr 20, 2021.

doi: 10.21037/jgo-21-78

View this article at: http://dx.doi.org/10.21037/jgo-21-78

^ ORCID: Zhikun Liang, 0000-0002-4417-6011; Xiaoyan Li, 0000-0003-3145-0517. 


\section{Introduction}

Since the cloning and reporting of the first member of the chemokine-like factor (CKLF)-like MARVEL transmembrane domain-containing (CMTM) family in 2001 (1), continuous efforts have been put into investigating the biological functions of this gene family in malignancies. The structural characteristics of the encoded proteins of the CMTM family have similarities with those of chemokines and transmembrane-4 superfamily (TM4SF) members. Previous research showed that CMTM family members may have involvement in the progression of autoimmune (2) and cardiovascular diseases (3). Besides, mounting studies have pointed toward CMTM family members being pivotal players in tumor-related biological processes (BP). Differential expression of CMTM genes has been described between tumor and normal samples, which suggests that CMTMs have considerable potential to serve as active regulators of tumor development in various cancers (4-10). In addition, the roles CMTM family proteins carry out in the progression, spread, and immunity of several types of cancer have been well studied (11-14). CMTM family proteins are also important mediators of tumor clinical characteristics. For instance, they can exacerbate chemoinsensitivity in non-small cell lung cancer (NSCLC) (15), and affect the prognosis of multiple cancers.

Gastric cancer (GC) is a major contributor to global malignancy-related mortality (16). Standard therapies for GC include surgical resection, chemotherapy, and chemoradiation in suitable cases (17); however, advanced cancers and metastasis are still a huge barrier to be overcome in the treatment of the disease (18). It is well known that a signature gene expression pattern could be utilized as a valuable biomarker to guide targeted therapy for GC. By combining global recognition of dysfunctional signaling pathways, the investigation of signature genes in cancer has the potential to support the individualization of cancer therapy. Analysis of molecular signatures and clinical parameters has shown there to be many different signature genes that can serve as valuable factors in GC patient stratification; however, the specific roles of the CMTM family remain to be elucidated.

Although some members in CMTM family have been revealed to involve in tumor-related molecular pathway, the molecular details and prognostic values of the entire CMTM gene family remained to be interpreted. As far as we know, a comprehensive bioinformatics analysis method for exploration of the potential prognostic value of CMTM proteins in GC has yet to be presented. A study focusing on the correlation between CMTM genes expression and prognosis of gastric cancer patients may shed light on the clinical value of CMTM family genes especially in the areas of gastric cancer early diagnosis and targeted therapy selection. Novel tumor biomarkers that can be used to diagnose or as prognostic indicators of GC are urgently required to improve patient outcomes and facilitate effective individualized therapeutic management. It is therefore important to perform an in-depth study of all CMTM genes in GC in order to definitively improve our understanding of the molecular mechanisms of GC progression and bolster the discovery of novel prognostic and therapeutic targets.

During this study, we performed a systematic bioinformatics analysis of different CMTM family genes and their related clinical parameters in public databases, focusing on the expressive patterns, underlying biological functions, and prognostic values of individual CMTM genes in GC. Moreover, in our analysis, we also studied the predicted functions and signaling pathways of CMTM mutations and their co-expressed genes. Additionally, an unsupervised hierarchical clustering analysis of a cohort of patients with stomach adenocarcinoma (STAD) from The Cancer Genome Atlas (TCGA) was performed. We identified 2 major subgroups: the first with CMTM1, CMTM2, CMTM3, CMTM5, and CMTM7, and the other with CMTM4, CMTM6, and CMTM8. The former group displayed decreased overall survival (OS) compared with the latter group, and thus, its signature (CMTM1, CMTM2, CMTM3, CMTM5, and CMTM7) was indicated as being related to a poor prognosis of GC.

We present the following article in accordance with the Materials Design Analysis Reporting (MDAR) reporting checklist (available at http://dx.doi.org/10.21037/jgo-21-78).

\section{Methods}

This study was carried out with adherence to the stipulations of the Declaration of Helsinki (as revised in 2013). As the study did not include any experiments involving animal or human subjects performed by any of the authors, neither ethical approval nor patient consent were required.

\section{Oncomine}

Oncomine database (https://www.oncomine.org/resource/ login.html) was employed for the analysis of the expression 
levels of different CMTM family genes in various cancers. For the statistical analysis, Student's $t$-test was used to verify expression differences. The threshold was set as a $\mathrm{P}$ value $<0.01$ and $\log 2$ fold change $>2$.

\section{GEPIA2}

Data of expression levels of CMTM family genes in GC were obtained from the GEPIA2 (Gene Expression Profiling Interactive Analysis) database (http://gepia2. cancer-pku.cn/). GEPIA2 is an online resource containing RNA sequencing (RNA-Seq) data of 9,736 tumors and 8,587 normal samples from The Cancer Genome Atlas (TCGA) and Genotype-Tissue Expression (GTEx) project, all of which were subjected to analysis according to standard processing pipelines (19). The RNA-Seq datasets were mainly downloaded from the UCSC Xena project (http://xena.ucsc.edu). Next, the differential expression of individual CMTM family genes between GC and healthy normal tissues was calculated. Statistical analysis was performed using the limma (20) package of $\mathrm{R}$ software. The calculation of $\mathrm{P}$ values was based on the Wilcoxon test, with the threshold set as a $\mathrm{P}$ value $<0.01$ and $\log 2$ fold change $>2$ to determine the statistically significant differences in the expression levels of target signature genes in tumor and normal samples.

\section{Association of CMTM family proteins with clinicopathological features of GC}

UALCAN (http://ualcan.path.uab.edu/) is an online public resource providing comprehensive cancer transcriptome data including from the TCGA and MET500. With UALCAN, the user can carry out tumor biomarker identification and verify genes that are potentially of interest. In the present research, we comprehensively analyzed the correlation between the expression of 8 CMTM family genes in GC, and clinical and pathological parameters. The methods used for statistical analysis were similar or identical to those mentioned above. Student's $t$-test was used to verify expression differences. The threshold was set as a $\mathrm{P}$ value $<0.01$. Due to the lack of conventional clinical information in UALCAN datasets, we additionally downloaded corresponding data of clinical parameters of GC patients from the TCGA database. Patients with incomplete data such as those lacking attribute values or with missing values within the records were deleted from the dataset. Thereafter, the remaining tumor samples with complete clinicopathological information were used to explore the statistically significant correlations of CMTM family protein levels with a variety of clinical features. The survival (https://cran.r-project.org/web/ packages/survival/index.html) package in $\mathrm{R}$ was used to perform the analysis.

\section{Human Protein Atlas}

Using the Human Protein Atlas (https://www.proteinatlas. org/), a web-based database that includes transcriptomic and immunohistochemical profiling data of 17 specific cancers from at least 8,000 patients (21), we obtained the immunohistochemical images of different CMTM family genes in both GC and normal tissues in order to verify the protein expression level of each CMTM family member. Therefore, the protein expression patterns could be analyzed in depth.

\section{Kaplan-Meier plotter}

Kaplan-Meier plotter (http://kmplot.com/analysis/) is a web-based database which has the capability to analyze the survival effects of 54,000 genes in 21 different cancers. This database was used to evaluate the effect of individual CMTM family genes on the prognosis of GC, of which it contains 1,440 samples. The median expression value of each CMTM family member in GC was set as the threshold to stratify patients into low- and high-expression groups. During survival curve plotting, hazard ratios (HRs) with $95 \%$ confidence intervals (CIs) and log-rank $\mathrm{P}$ values were simultaneously computed for statistical significance analysis. The association between the expressions of target genes and survival in GC was analyzed using the Kaplan-Meier method followed by the log-rank test to calculate the $\mathrm{P}$ value. The threshold for statistical significance was set as a $\mathrm{P}$ value $<0.05$.

\section{TCGA and cBioPortal}

The Cancer Genome Atlas (https://www.cancer.gov/tcga) database aims to understand the molecular basis of cancer by characterizing different aspects of the cancer genome, including copy number, epigenetic alterations, mutations, and gene expression. As a landmark global cancer genome project, the TCGA database offers RNA-seq data with complete clinical data of both tumor and normal tissue, covering 33 cancer types. The cBio Cancer Genomics 
Portal (cBioPortal) represents a pivotal online platform with open-access for visualization and analysis of over 5,000 tumor samples from 105 cancer studies $(22,23)$. A STAD (TCGA, Firehose Legacy) dataset (24) was selected for analysis of the mutational profiles of CMTM family members in GC, as well as the co-expression correlations of each CMTM family member. Analysis of the coexpression associations among the 8 CMTM genes was performed with the Corrplot package (https://cran.r-project. org/web/packages/corrplot/index.html) of R software, and Spearman's correlation was adopted to calculate the difference significance index. A statistically significant difference was defined as $\mathrm{P}$ value $<0.05$. The FactoMineR package in $\mathrm{R}$ studio was used to perform principal component analysis (PCA) and unsupervised hierarchical clustering (HC). Additionally, HCPC in the FactoMineR package was used to determine the number of clusters. After patient clustering, an analysis of survival was performed using the survival and SurvMiner packages. The cluster with the highest relative loss of inertia was identified as the default partition in 3 clusters. Major clusters \#1 and \#2 were used for subsequent analysis, while cluster \#3 was excluded due to the low number of cases (only 1 patient).

\section{Gene Ontology (GO) and Kyoto Encyclopedia of Genes and Genomes pathway analyses (KEGG)}

The functional enrichment of CMTM family members and their related co-expressed genes was examined through GO and KEGG analyses performed in the Database for Annotation, Visualization and Integrated Discovery (DAVID, https://david.ncifcrf.gov/) $(25,26)$. In the GO analysis, genes were analyzed with respect to 3 aspects: $\mathrm{BP}$, cellular components (CC), and molecular functions (MF). To illuminate the molecular mechanisms underlying the involvement of the CMTM family in GC, we also performed a protein-protein interaction (PPI) network analysis using the STRING database (http://string-db.org/).

\section{Statistical analysis}

Chi-square, ANOVA, and Student $t$-test statistical analyses were conducted based on R script. All analysis results were considered to be statistically significant at $\mathrm{P}$ value $<0.05$. The GEPIA and Oncomine databases also provided $t$-test analysis. Kaplan-Meier plotter online tool was applied for statistical analyses of hazard ratios and survival rates. A logrank test was performed to evaluate the equality of survival curves between different patient groups.

\section{Results}

\section{CMTMs at the transcriptional level in patients with GC}

Using the Oncomine and GEPIA2 databases, we identified 8 CMTM family members in a variety of tumors, including GC. The results of the Oncomine database analysis revealed CMTM family genes to be highly expressed in multiple tumors (Figure 1), including 2 family members which were differentially expressed in GC. With respect to CMTM3, a high expression level was found in both the Wang Gastric dataset $(\mathrm{FC}=2.130, \mathrm{P}=3.69 \mathrm{E}-5)$ and the Cho Gastric dataset ( $\mathrm{FC}=8.470, \mathrm{P}=1.83 \mathrm{E}-4)$. We also identified the upregulation of CMTM3 in the Derrico Gastric dataset $(\mathrm{FC}=5.055, \mathrm{P}=2.39 \mathrm{E}-6)$. More specifically, the comparison between diffuse gastric adenocarcinoma and normal samples showed that CMTM3 was significantly upregulated in the tumor tissues $(\mathrm{FC}=2.562, \mathrm{P}=4.00 \mathrm{E}-3)$, while CMTM3 was highly expressed in intestinal-type GC compared with normal samples $(\mathrm{FC}=2.690, \mathrm{P}=1.47 \mathrm{E}-5)$ in the Derrico Gastric dataset. Contrastingly, we found low expression of CMTM4 in multiple datasets, including the Allander Gastric dataset $(\mathrm{FC}=-3.026, \mathrm{P}=1.84 \mathrm{E}-5)$, Wang Gastric dataset (FC $=-2.193, \mathrm{P}=3.24 \mathrm{E}-4)$, Derrico Gastric dataset $(\mathrm{FC}=-3.211, \mathrm{P}=1.41 \mathrm{E}-4)$, and Cho Gastric dataset (intestinal-type adenocarcinoma vs. normal: $\mathrm{FC}$ $=-2.336, \mathrm{P}=4.27 \mathrm{E}-5$, diffuse type $v s$. normal: $\mathrm{FC}=-2.157$, $\mathrm{P}=1.56 \mathrm{E}-4)$. However, no significant differential expression of the other CMTM family members was identified over the Oncomine GC datasets. To verify these findings, further analysis of the transcript levels of the 8 CMTM family genes was performed using the GEPIA2 database, which contains RNA-seq data and clinical information of over 31 different cancers (Figure 2). The mRNA expression levels of CMTM1/3/6/7/8 were demonstrated to be significantly upregulated in GC tissues compared with healthy samples, whereas no statistically significant difference was identified for the expression of CMTM2/4/5.

\section{Relationship between CMTM family members and the clinicopathological parameters of GC}

Using the UALCAN database (TCGA data were processed by unified standards), the associations between the expression levels of 8 CMTM family genes and the clinical features of patients with GC were further analyzed, 

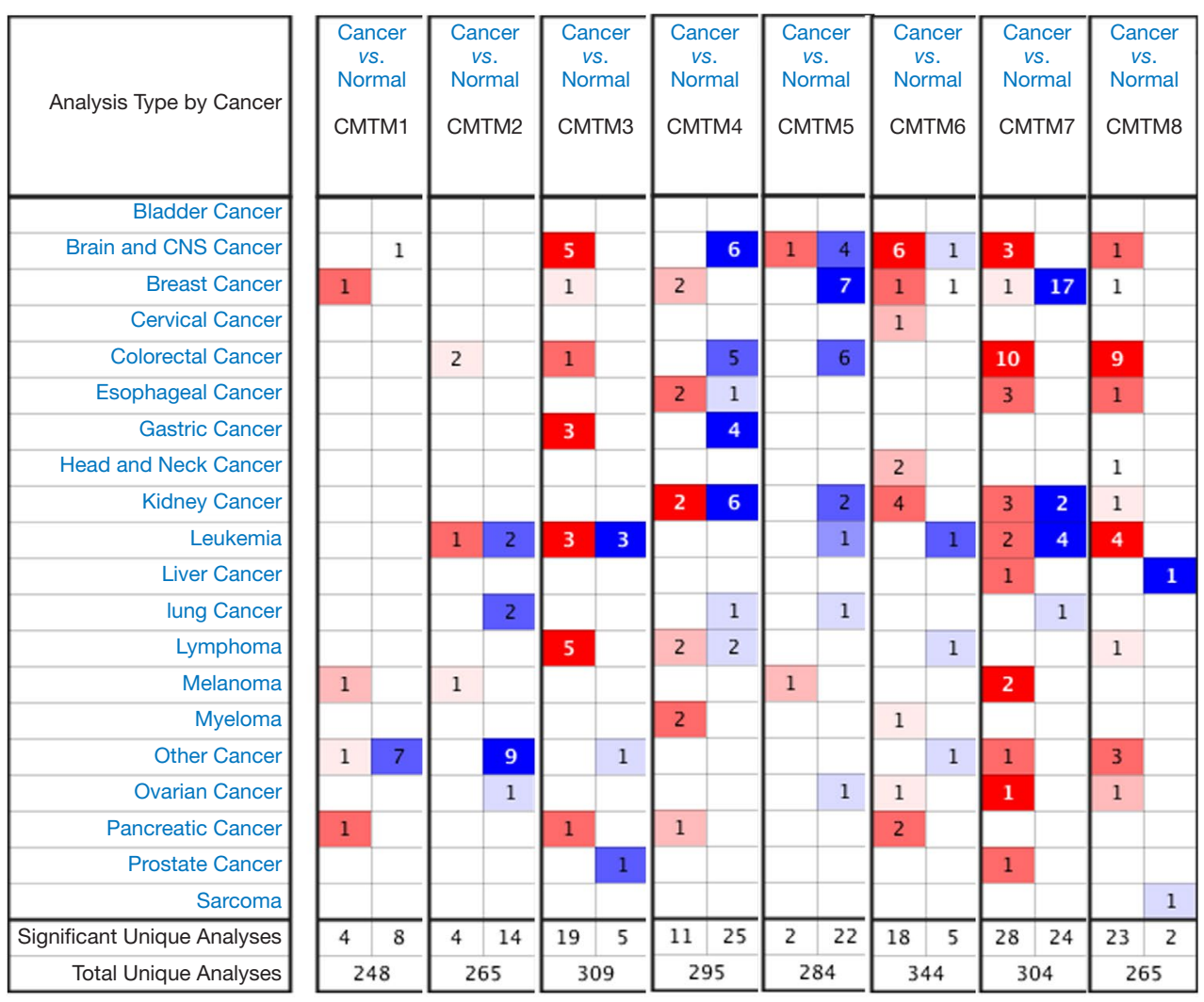

Figure 1 The expression levels of CMTMs in 20 different cancer types (Oncomine). Student's $t$-test was applied to compare the differences of expression levels. The threshold was defined as $\mathrm{P}<0.05$ and fold change: 1.5 . The gene rank was set as "all". CMTM genes, chemokinelike factor-like MARVEL transmembrane domain-containing family genes.

and the results are shown in Figures 3 and 4. All selected gastric cancer patient samples reported clinical parameters included cancer stage, tumor grade, lymph node metastasis status, and $H$. pylori status. $42.4 \%$ of patients (188 of 443 ) reported available data regarding $H$. pylori status. Of these, $37.9 \%$ of patients showed no $H$. pylori infection whereas $4.5 \%$ of patients reported $H$. pylori infection. Lymph node metastases were identified in $65.9 \%$ patients (292 of 443), involving $26.8 \%$ patients with N1 stage, $19.1 \%$ patients with $\mathrm{N} 2$ stage, $7.2 \%$ patients with N3 stage, $11.0 \%$ patients with $\mathrm{N} 3 \mathrm{a}$ stage and $1.5 \%$ patients with N3b stage. For distal metastasis, $6.8 \%$ of patients (30 of 443) reported cancer metastasis. The distribution of gastric cancers by tumor stage was: T1, 5.1\%; T2, 20.9\%; T3, 44.6\%; and T4, 26.8\%, whereas tumor grade distribution was: G1, 2.7\%; G2, 35.8\%; and G3, 59.3\%. The ten most frequently mutated genes in selected TCGA samples were: TP53, 48.1\%; LRP1B, 26.3\%; ARID1A, $25.8 \%$; FAT4, 21.5\%; PCLO, 20.3\%; KMT2D, $17.5 \%$;
PIK3CA, 16.5\%; ACVR2A, 14.9\%; LRRK2, 14.9\%; and KMT2C, $13.9 \%$. A statistically significant correlation was found between the expression of CMTM family genes and most clinicopathological features of GC. The expression of half of the CMTM family members (CMTM1/3/6/7) was associated with a more advanced tumor stage. In contrast, tumors with CMTM2/5/8 expression tended to be of a lower stage. Meanwhile, no statistically significant relationship was discovered between CMTM4 expression and clinicopathological parameters of GC.

\section{Protein expression of the CMTM family}

We analyzed the protein expressions of CMTM family genes in GC using the Human Protein Atlas, and the results were shown in Figure 5. The protein expression of CMTM1/3/6 was upregulated in tumor samples, whereas CMTM4/5/8 protein was highly expressed in healthy tissue samples. However, we failed to find immunohistochemical 

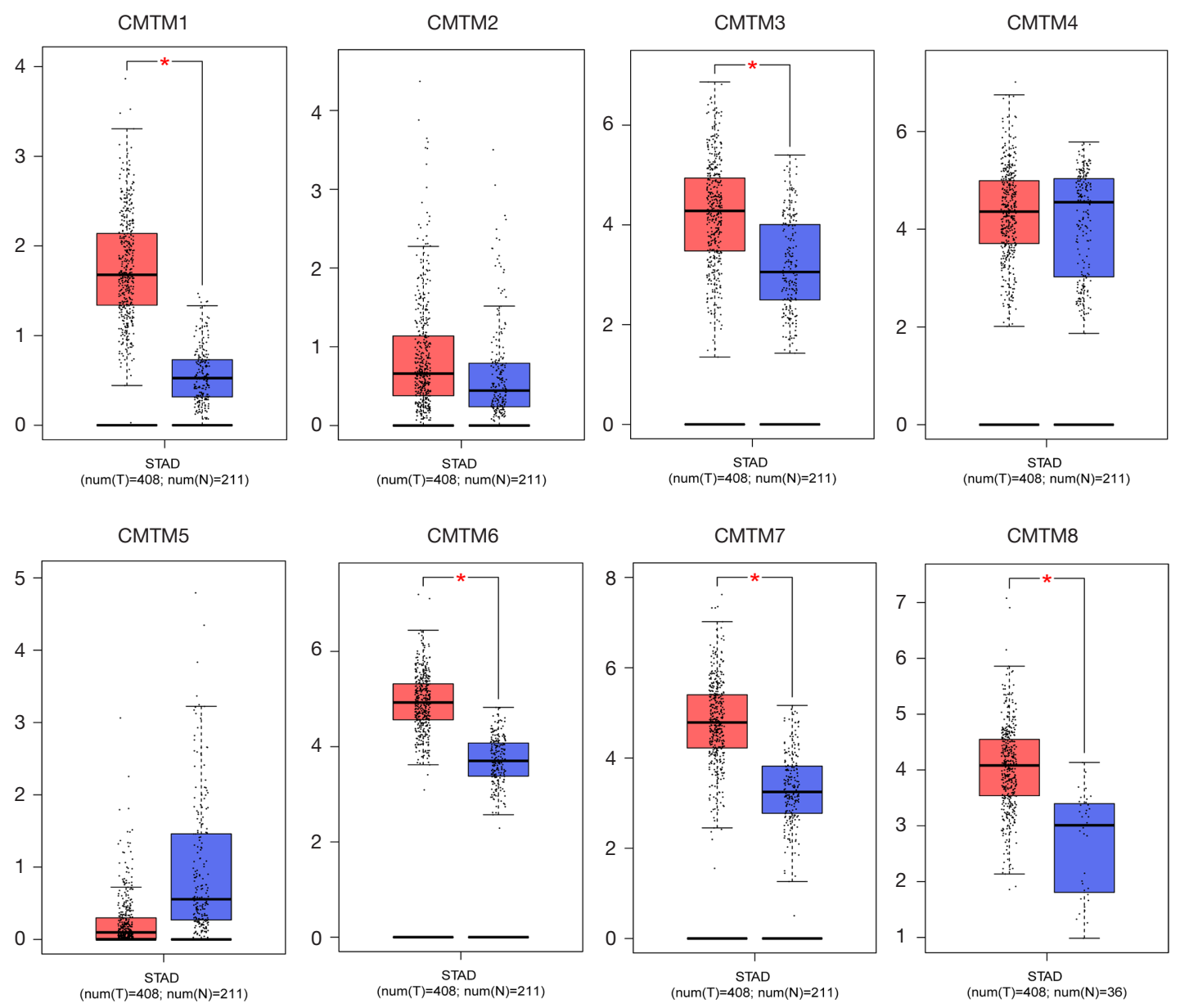

Figure 2 The CMTMs expression levels of gastric cancer in GEPIA2 database. The CMTMs expression levels of gastric cancer compared with those in normal healthy tissues (box plot). ${ }^{*}, \mathrm{P}<0.05$. STAD, stomach adenocarcinoma. CMTM genes, chemokine-like factor-like MARVEL transmembrane domain-containing family genes.

images of CMTM2 and CMTM7 on the Human Protein Atlas. Except for CMTM4, these results are in line with the outcomes of CMTM family mRNA expression analysis in the present study.

\section{Values of the CMTM family in prognosticating GC survival}

To determine the value of each CMTM family member as a prognostic factor, we applied the Kaplan-Meier plotter to calculate the statistical correlation between the expression of CMTM genes and the survival time of GC patients, and the results are shown in Figure 6. Significant correlations were found between most CMTM family members and GC prognosis. Kaplan-Meier curve analysis combined with the log-rank test indicated a significant correlation between upregulation of CMTM 3 or CMTM5 and poor overall survival (OS), progression-free survival (PFS), and post-progression survival (PPS) $(\mathrm{P}<0.05)$. High levels of CMTM2/4/6 mRNA were remarkably correlated with longer OS, PFS, and PPS. High CMTM1 or CMTM7 mRNA expression was significantly correlated with poor PPS, but not with PFS or OS. A high level of CMTM8 mRNA displayed a close association with longer OS, but not PFS or PPS.

\section{Gene mutations and co-expressed genes of the CMTM family}

Using cBioPortal, mutation analysis of CMTM the family 

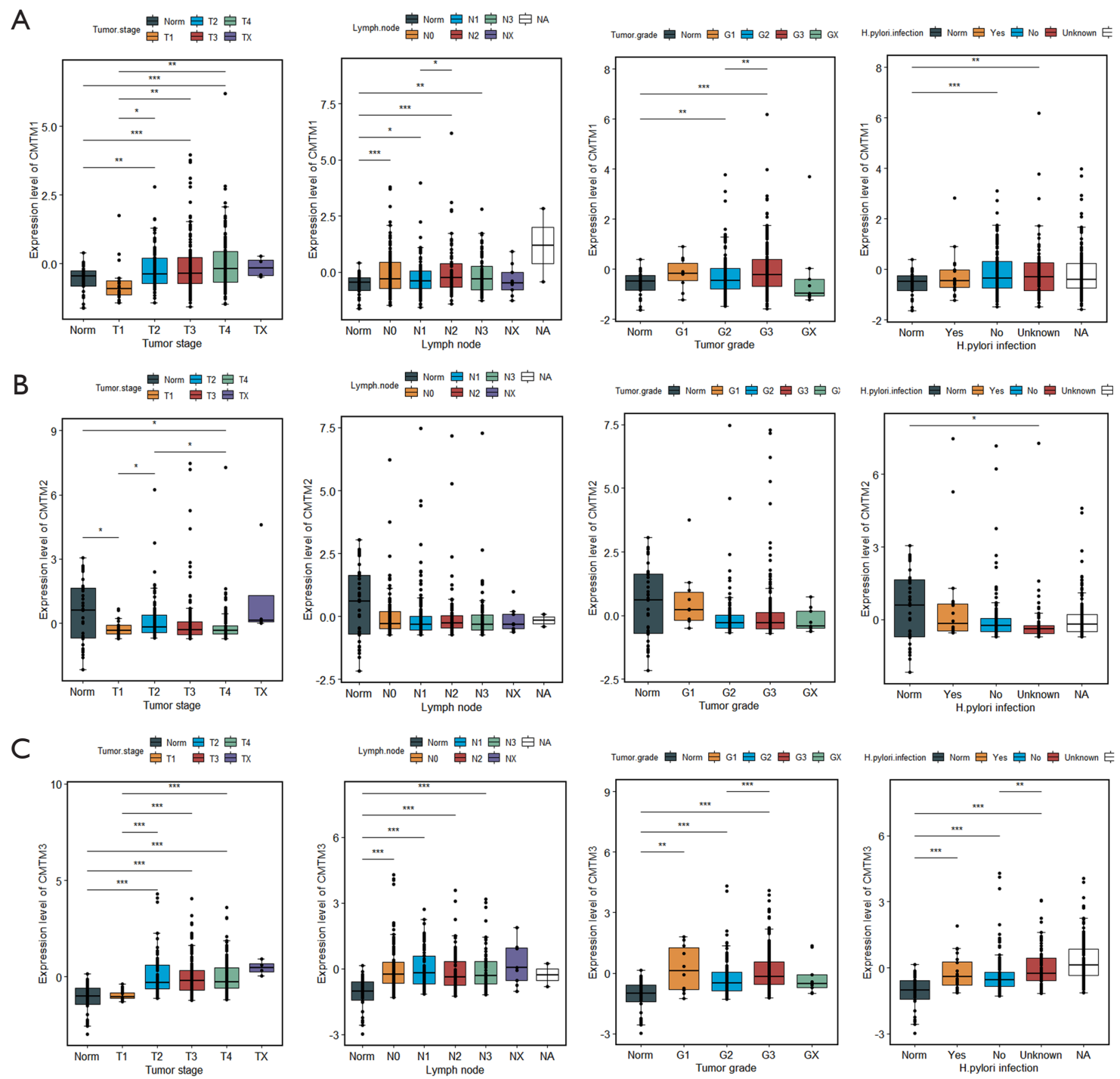

D
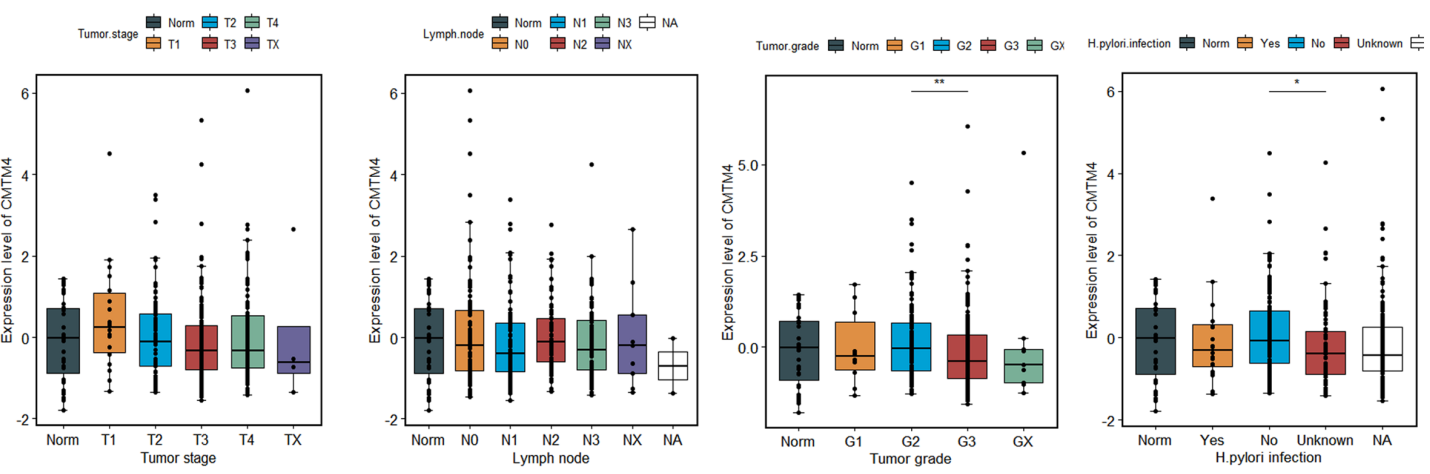

Figure 3 Correlation between CMTM1-4 expression and clinical parameters of gastric cancer. Clinical parameters included cancer stage, tumor grade, lymph node metastasis status, and H. pylori status. (A) Association of mRNA expression levels of CMTM1 with clinical parameters. (B) Association of mRNA expression levels of CMTM2 with clinical parameters. (C) Association of mRNA expression levels of CMTM3 with clinical parameters. (D) Association of mRNA expression levels of CMTM4 with clinical parameters. $\left(^{*}, \mathrm{P}<0.05\right.$; ${ }^{* *}, \mathrm{P}<0.01$; ***, $\mathrm{P}<0.001)$. Norm, normal. CMTM genes, chemokine-like factor-like MARVEL transmembrane domain-containing family genes. 

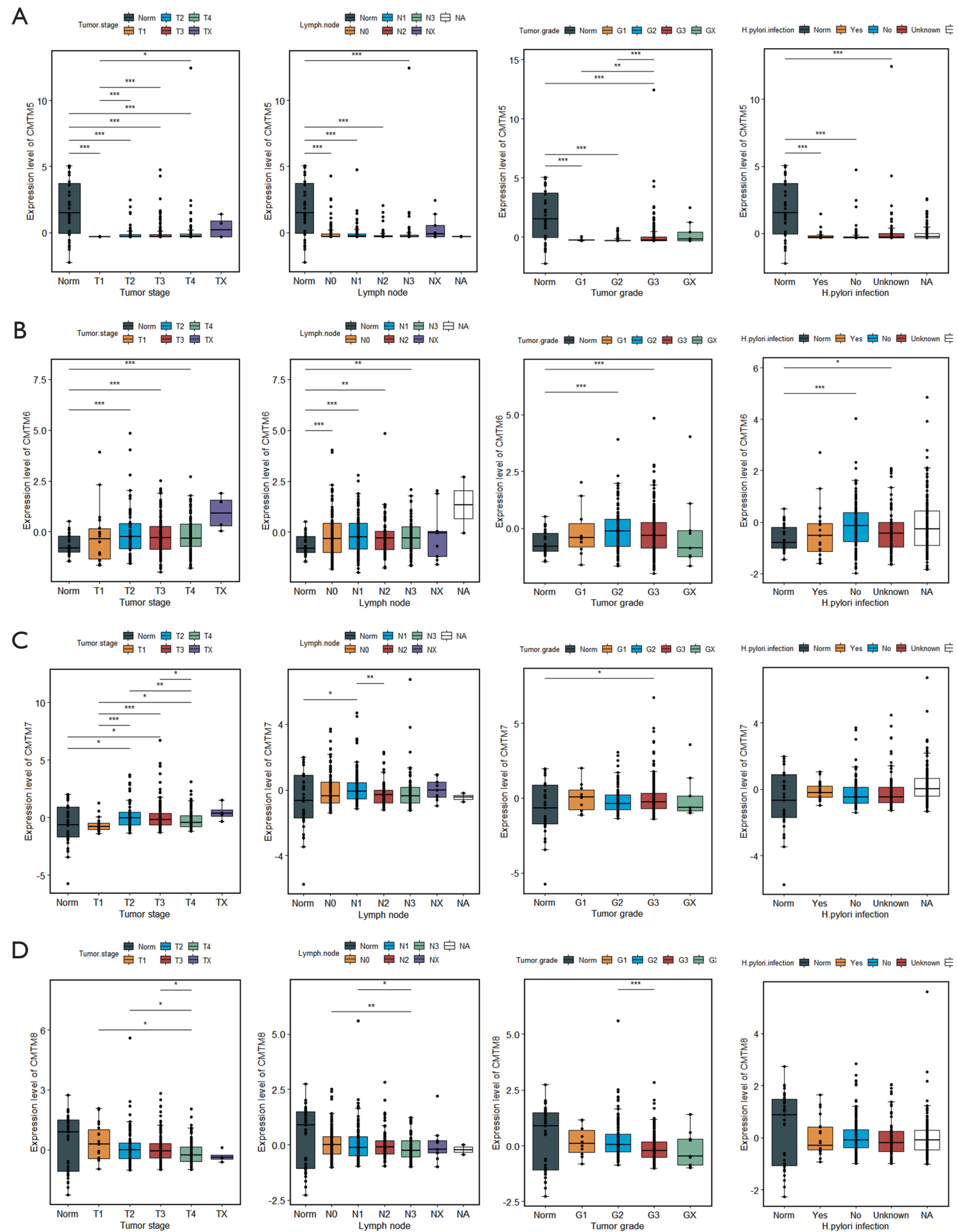

Figure 4 Correlation between CMTM5-8 expression and clinical parameters of gastric cancer. Clinical parameters included cancer stage, tumor grade, lymph node metastasis status, and H. pylori status. (A) Association of mRNA expression levels of CMTM5 with clinical parameters. (B) Association of mRNA expression levels of CMTM6 with clinical parameters. (C) Association of mRNA expression levels of CMTM7 with clinical parameters. (D) Association of mRNA expression levels of CMTM8 with clinical parameters. ${ }^{*}, \mathrm{P}<0.05$; ${ }^{* *}, \mathrm{P}<0.01$; ***, $\mathrm{P}<0.001)$. Norm, normal. CMTM genes, chemokine-like factor-like MARVEL transmembrane domain-containing family genes. 
A

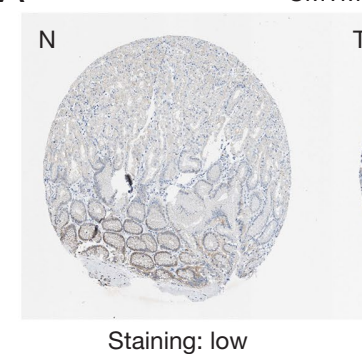

C

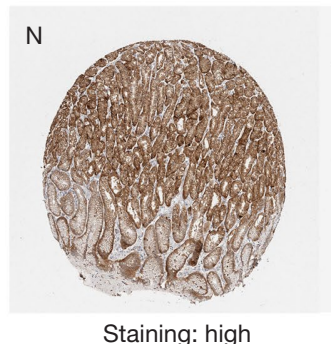

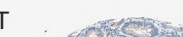

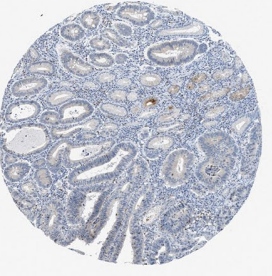

Staining: medium

CMTM4

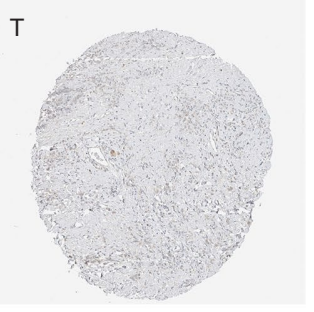

T

Staining: low

E

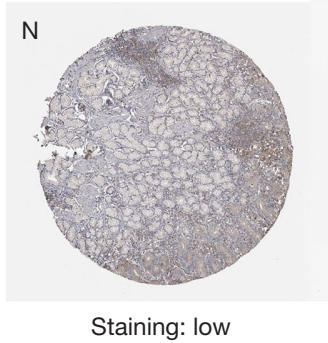

CMTM6

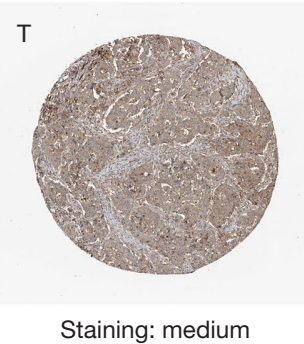

B

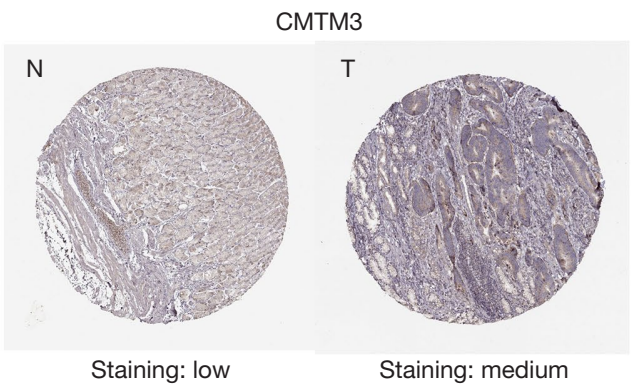

D

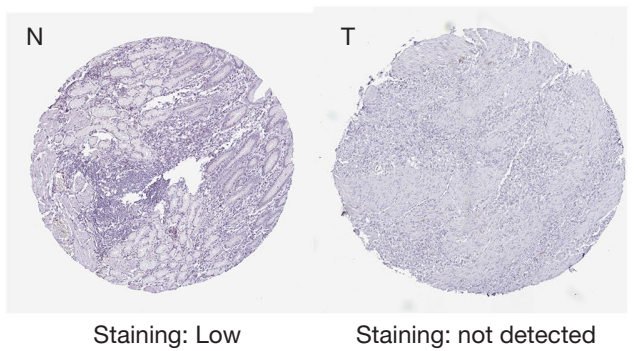

F

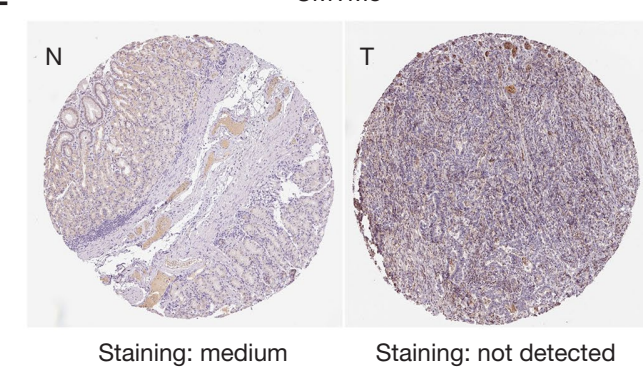

Figure 5 Immunohistochemical images of CMTM family members from the Human Protein Atlas database. (A) Immunohistochemical images of CMTM1 protein in tumor and normal tissues. (B) Immunohistochemical images of CMTM3 protein in tumor and normal tissues. (C) Immunohistochemical images of CMTM4 protein in tumor and normal tissues. (D) Immunohistochemical images of CMTM5 protein in tumor and normal tissues. (E) Immunohistochemical images of CMTM6 protein in tumor and normal tissues. (F) Immunohistochemical images of CMTM8 protein in tumor and normal tissues. The scale for each image is $200 \mu \mathrm{m}$. N, normal. T, tumor. CMTM genes, chemokine-like factor-like MARVEL transmembrane domain-containing family genes.

in GC was performed. The result showed that mutations of CMTM genes were observed in 194 out of 369 GC patients, with the mutation rate exceeding $53 \%$ (Figure 7). Furthermore, the associations among the CMTM family members were analyzed based on their mRNA expression levels. Pearson's correction was introduced into this calculation. As exhibited in Figure $7 B$, the most significant positive correlations (Pearson correlation $\geq 0.2, \mathrm{P}<0.05$ ) were: CMTM1 with CMTM6 (Pearson correlation $=0.21$, $\mathrm{P}<0.01$ ), CMTM3 with CMTM7 (Pearson correlation $=0.26, \mathrm{P}<0.01$ ), CMTM6 with CMTM7 (Pearson correlation $=0.56, \mathrm{P}<0.01)$, CMTM6 with CMTM7 (Pearson correlation $=0.61, \mathrm{P}<0.01)$, and $\mathrm{CMTM} 7$ with
CMTM8 (Pearson correlation $=0.55, \mathrm{P}<0.01$ ). The CMTM genes with the most significant negative correlations (Pearson correlation $\leq-0.2, \mathrm{P}<0.05$ ) were: CMTM3 with CMTM4 (Pearson correlation $=-0.31, \mathrm{P}<0.01$ ), and CMTM4 with CMTM5 (Pearson correlation $=-0.2$, $\mathrm{P}<0.01)$. Subsequently, the interconnections between CMTM family genes and their co-expressed genes were analyzed through the construction of a PPI network using the STRING database (Figure 7D). Subsequently, the corresponding biological functions of CMTMs and their co-expressed genes related to tumor progression were investigated using the DAVID database (Figure 8). In GO analysis, these genes were analyzed with respect to 3 aspects: 

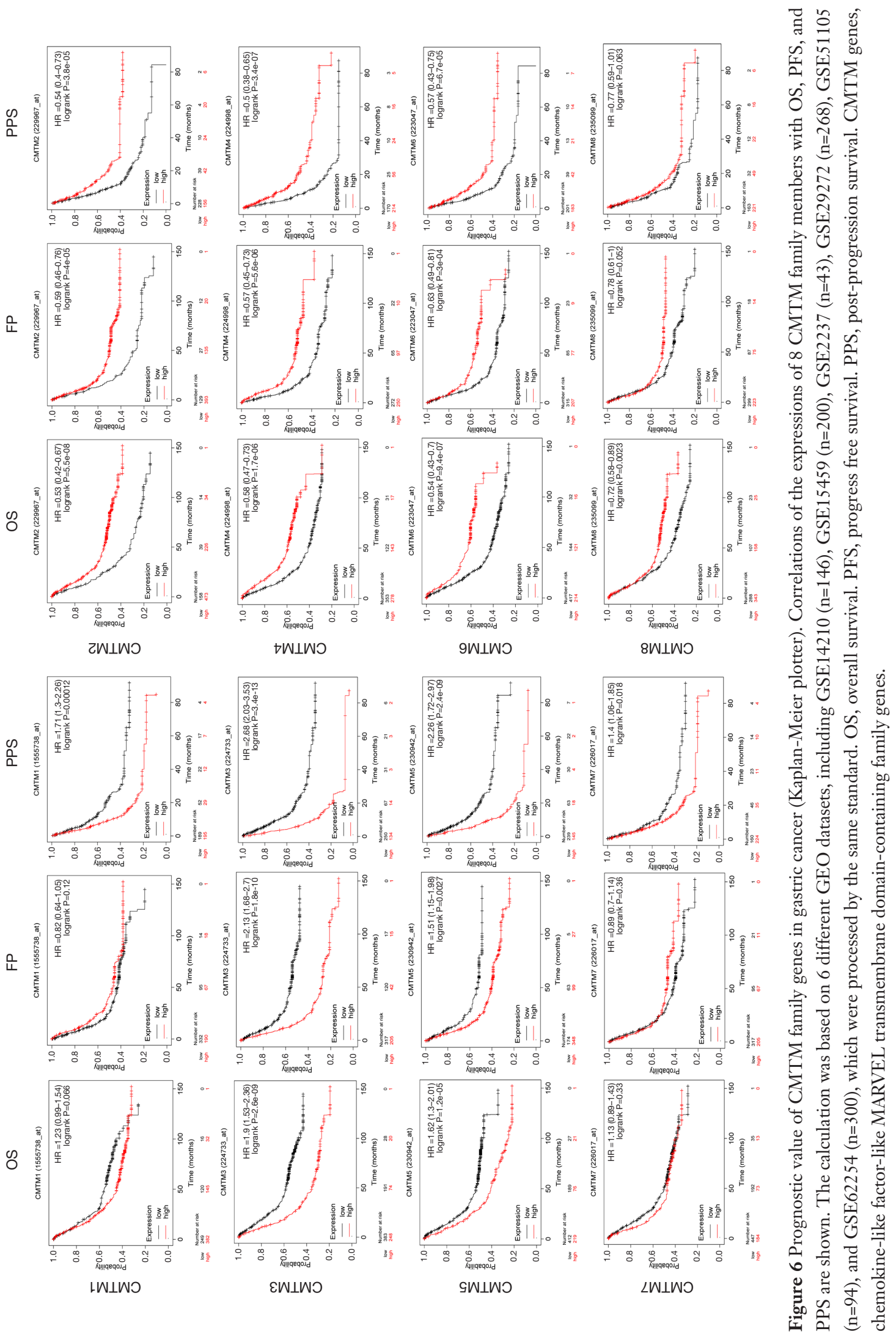

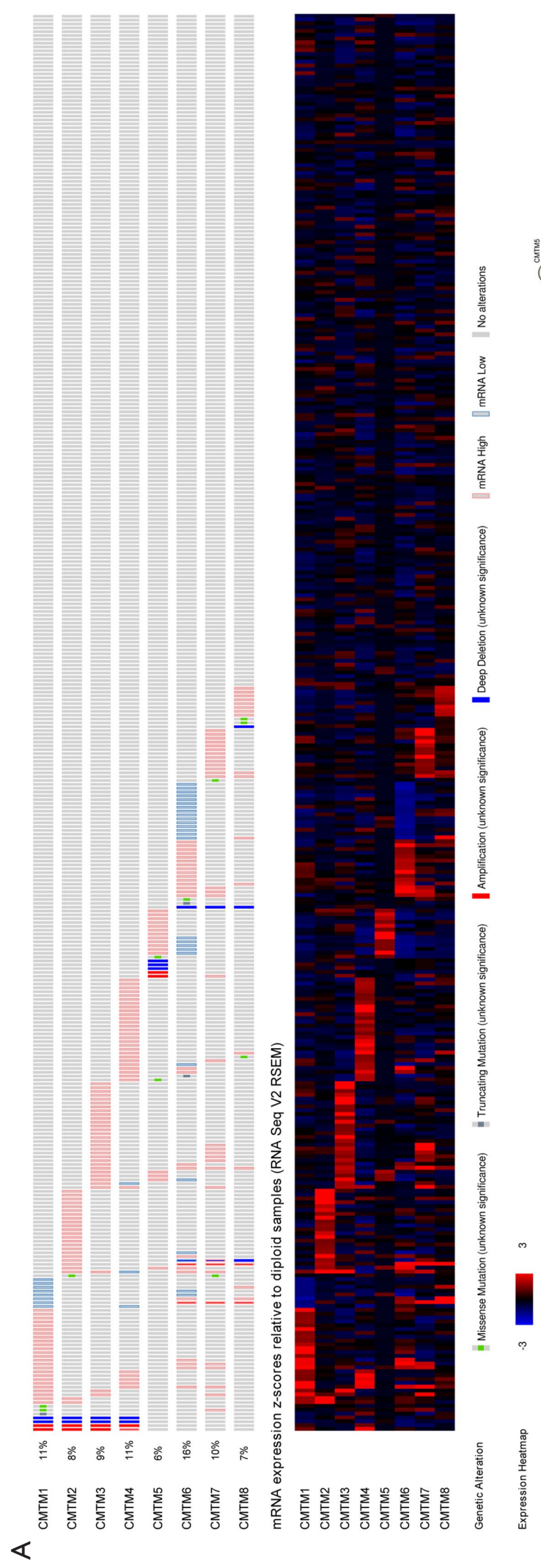

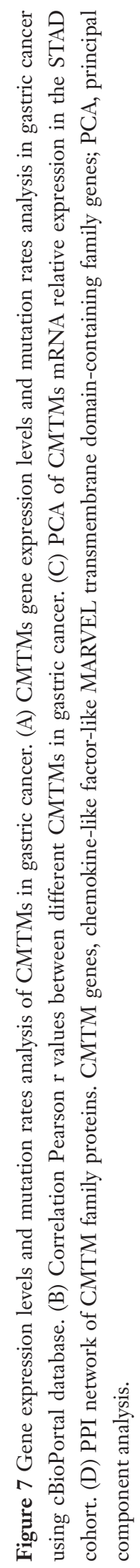

$\infty$

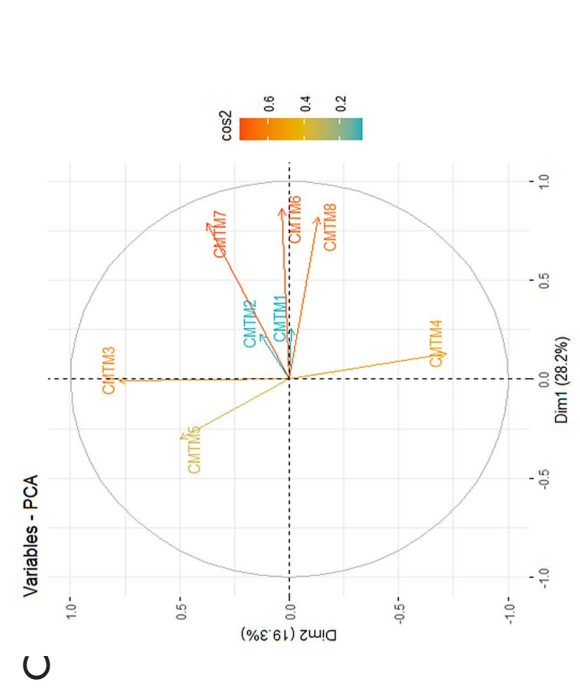

U

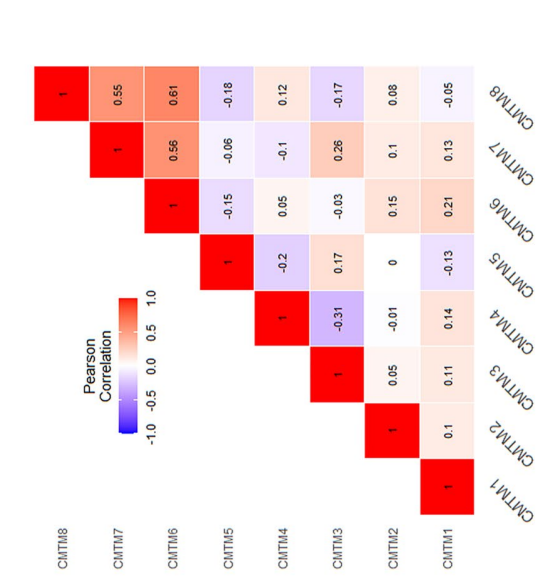

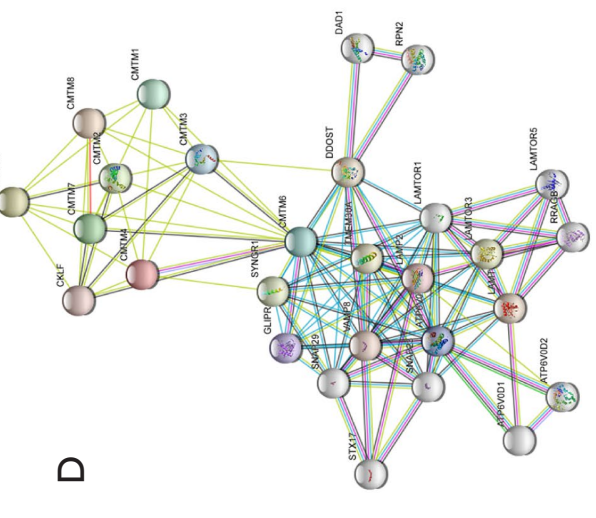

क्षे :

$\sum_{i=1}^{\infty} \sum_{0}^{ \pm}$

更

ङ. क

苛 $\sum_{i=1}^{\infty}$

.0

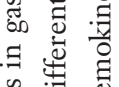

$\sum_{i}^{\infty} \overline{0}$

पे

点总芯

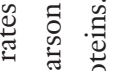

ㅇํㅇ

䒿 $\stackrel{\Xi}{\Xi}$

芩

兽

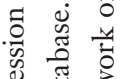

育 究

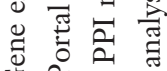

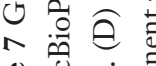

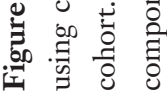


A

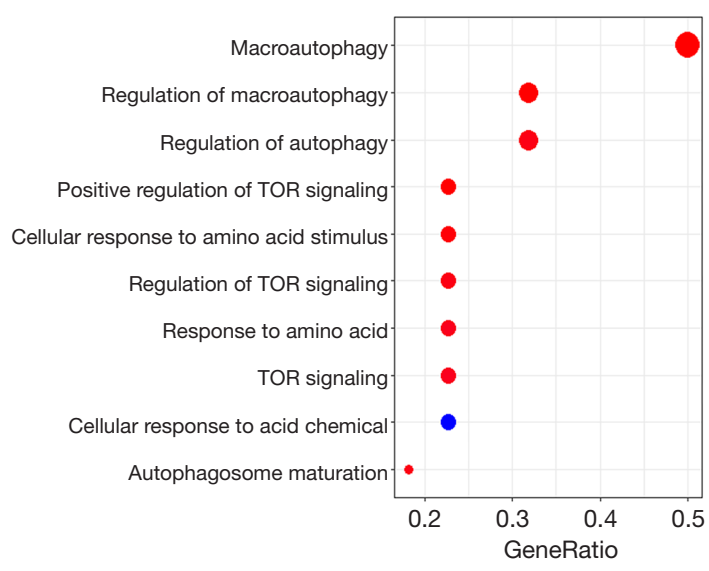

B

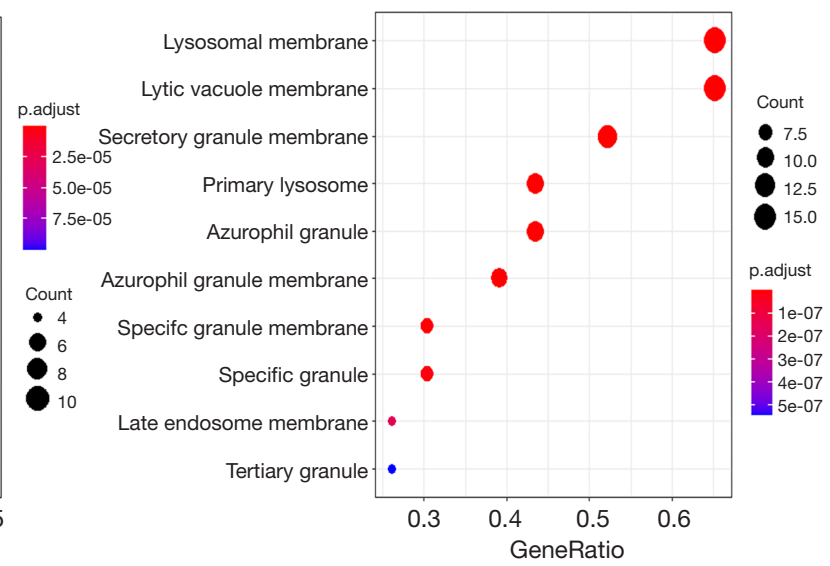

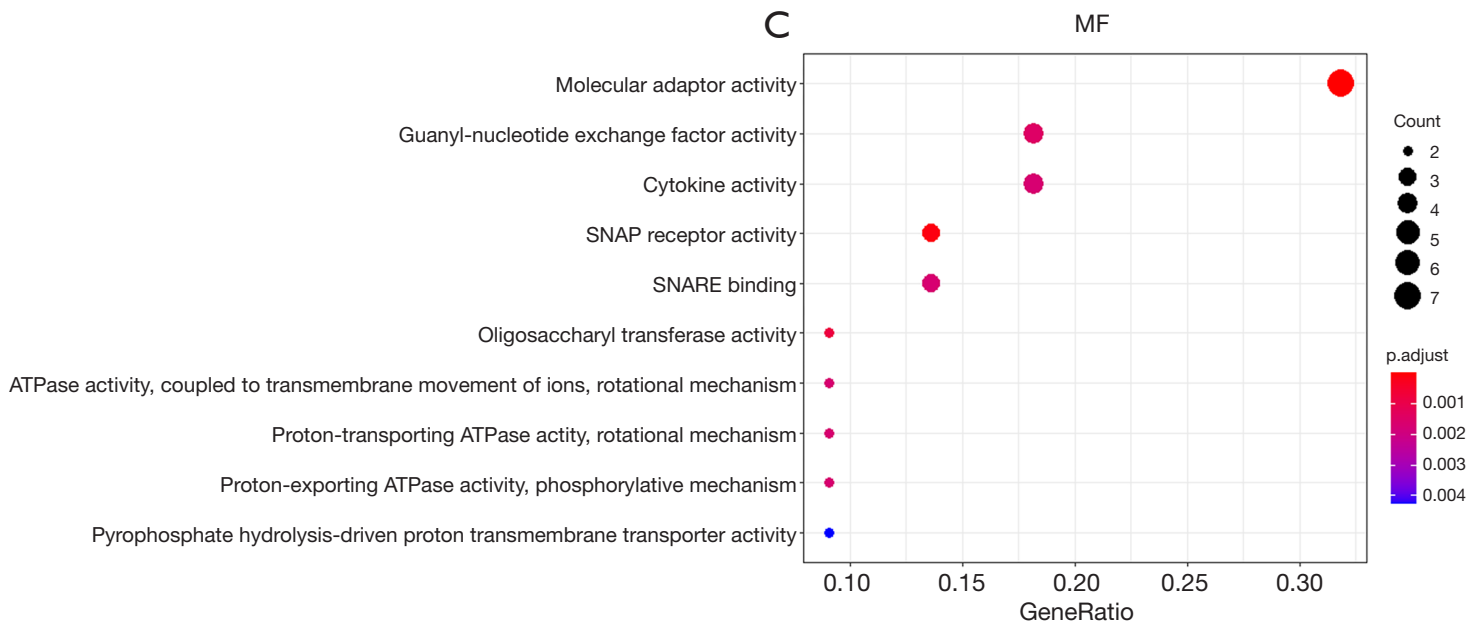

Figure 8 Results of functional enrichment of CMTM family genes and their related co-expressed genes in GO analysis. In GO analysis, all genes were analyzed with respect to biological processes BP, CC, and MF. CMTM genes, chemokine-like factor-like MARVEL transmembrane domain-containing family genes; GO, Gene Ontology; BP, biological processes; CC, cellular components; MF, molecular functions.

$\mathrm{BP}, \mathrm{CC}$, and MF. The results of functional annotation analysis suggested 8 CMTM family members and their related co-expressed genes to be significantly enriched in signaling pathways including macroautophagy, regulation of autophagy, TOR signaling, cytokine activity. The KEGG pathways related to the genes were further analyzed, and the results are shown in Table 1.

\section{Unsupervised hierarchical clustering of the STAD cohort}

For the purpose of stratifying patients with similar CMTM expression characteristics, we applied an algorithm of unsupervised hierarchical clustering to analyze the STAD cohort data. As a result, 3 different groups of patients were successfully achieved, as shown on the dendrogram in Figure 9A. Cluster \#1 (red) contained 232 patients and cluster \#2 (yellow) contained 153 patients. We also obtained a group that contained only 1 patient (cluster \#3). Thus, we focused on the in-depth analysis of clusters \#1 and \#2. Cluster \#1 was distinguished by CMTM4/6/8 upregulation, while cluster \#2 was characterized by extensive upregulation of CMTM1/2/3/5/7 (Figure 9B). Except for that of CMTM6, these differential expressions were calculated to be statistically significant (all $\mathrm{P}<0.05$ ). Additionally, on the Kaplan-Meier plot, cluster \#2 was found to be correlated with poorer OS than cluster \#1 (Figure 9C). The clinicopathological characteristics of the patients in 2 different clusters were also analyzed, and the results are 
Table 1 The functions of CMTM family members and genes significantly associated with CMTM alterations in gastric cancer predicted through KEGG pathway analysis

\begin{tabular}{lllc}
\hline Category & Term & Count & Adjusted P value \\
\hline KEGG & hsa04130-SNARE interactions in vesicular transport & 4 & $* * *$ \\
KEGG & hsa04140-Autophagy-animal & 5 & $* * *$ \\
KEGG & hsa04150-mTOR signaling pathway & 5 & $* *$ \\
KEGG & hsa04966-Collecting duct acid secretion & 2 & $*$ \\
KEGG & hsa04142-Lysosome & 3 & $*$ \\
KEGG & hsa00513-Various types of N-glycan biosynthesis & 2 & $*$ \\
KEGG & hsa04145-Phagosome & $*$ \\
KEGG & hsa00510-N-Glycan biosynthesis & 3 & $*$ \\
KEGG & hsa05110-Vibrio cholerae infection & 2 & $*$ \\
KEGG & hsa05152-Tuberculosis & 2 & 0.012 \\
KEGG & hsa05120-Epithelial cell signaling in Helicobacter pylori infection & 3 & 0.013 \\
KEGG & hsa04721-Synaptic vesicle cycle & 2 & 0.017 \\
KEGG & hsa05323-Rheumatoid arthritis & 2 & 0.027 \\
KEGG & hsa04611-Platelet activation & 2 & 0.029 \\
KEGG & hsa00190-Oxidative phosphorylation & 2 & 0.043 \\
KEGG & hsa04141-Protein processing in endoplasmic reticulum & 2 & $*$
\end{tabular}

${ }^{*} \mathrm{P}<0.01 ;{ }^{* \star} \mathrm{P}<0.001 ;{ }^{* \star *} \mathrm{P}<0.0001$. CMTM, chemokine-like factor (CKLF)-like MARVEL transmembrane domain-containing; KEGG, Kyoto Encyclopedia of Genes and Genomes.

shown in Figure 10. We found an obvious trend of tumor grade $(\mathrm{G} 3)$ enrichment in cluster \#2 $(\mathrm{P}<0.05)$; however, differences in other clinicopathological features including tumor stage, metastatic lymph node status, and H. pylori status did not reach statistical significance. Therefore, the CMTM expression pattern might have the potential to be a valuable prognostic biomarker of GC.

\section{Discussion}

The CMTM family and its pathway members have previously been implicated in the regulation of disease development in various cancers, including GC. However, the individual roles of the CMTM genes have remained unclear. The chromosomal locations of genes in the CMTM family differ as follows: CMTM1-4 (chromosome 16q), CMTM5 (chromosome 14q11.2), and CMTM6-8 (chromosome 3p22.3) (27). Different alternative RNA splicing forms of CMTM genes produce CMTM protein isoforms in different organs and cellular locations, including cytomembrane and cytoplasm. Analysis of CMTM family genes and their encoded gene products provides insights into the functional characteristics of this community and reveals their unique association with classical chemokines and transmembrane 4 super family (TM4SF) members. The MARVEL domain of CMTM proteins, which has 4 transmembrane helix-architecture, is associated with biological functions relating to vesicle transport and cellular membrane binding $(2,28,29)$. To the best of our knowledge, this is the first time that bioinformatics methods have been applied to study the CMTM family with respect to their mRNA expression level, mutation status, and prognostic value in GC. This approach might help to advance our molecular understanding of GC, uncover new treatment targets, and more accurately predict the prognosis of patients with GC.

CMTM1, which consists of 23 isoforms (CMTM1 v1$\mathrm{v} 23$ ), is located at chromosome 16q21. A previous study indicated that CMTM1-v17 proteins encoded by open reading frame 2 were upregulated in various cancers, 

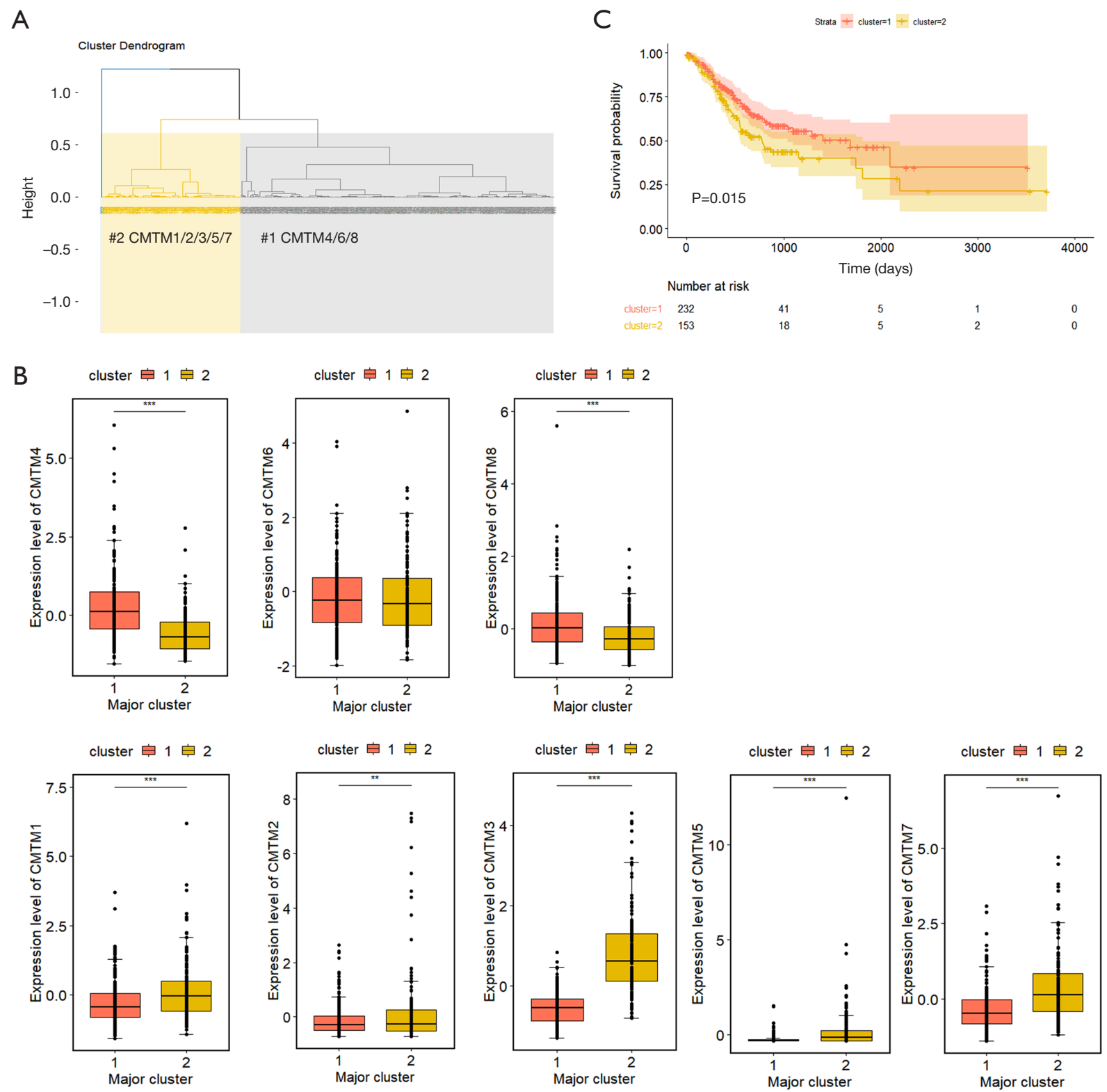

Figure 9 An algorithm of unsupervised hierarchical clustering was applied to analysis the STAD cohort data based on the CMTMs gene expression levels. (A) Two different groups of patients were successfully achieved, as shown on the dendrogram: Cluster \#1 contained 232 patients and cluster \#2 contained 153 patients. (B) The CMTMs gene expression levels in clusters \#1 and \#2 were shown by boxplot. An unpaired t-test was applied to compare the differences in expression levels (***, $\mathrm{P}<0.001$; **, $\mathrm{P}<0.01)$. (C) The Kaplan-Meier curves of clusters \#1 and \#2. STAD, stomach adenocarcinoma. CMTM genes, chemokine-like factor-like MARVEL transmembrane domaincontaining family genes.

including breast, kidney, lung, ovarian, and liver cancer (30). The same investigation also verified the involvement of CMTM1-v17 in the regulation of cellular proliferation and tumor necrosis factor- $\alpha$ (TNF- $\alpha)$-induced apoptosis in breast cancer through activation of the nuclear factor kappa-B (NF- $\kappa B)$ pathway. Si et al. reported CMTM1-v17 expression to be higher in non-small cell lung carcinoma samples compared to adjacent normal tissues, which might lead to a poor prognosis due to enhanced chemoresistance (15). To date, the role of CMTM1 in GC has remained elusive. 

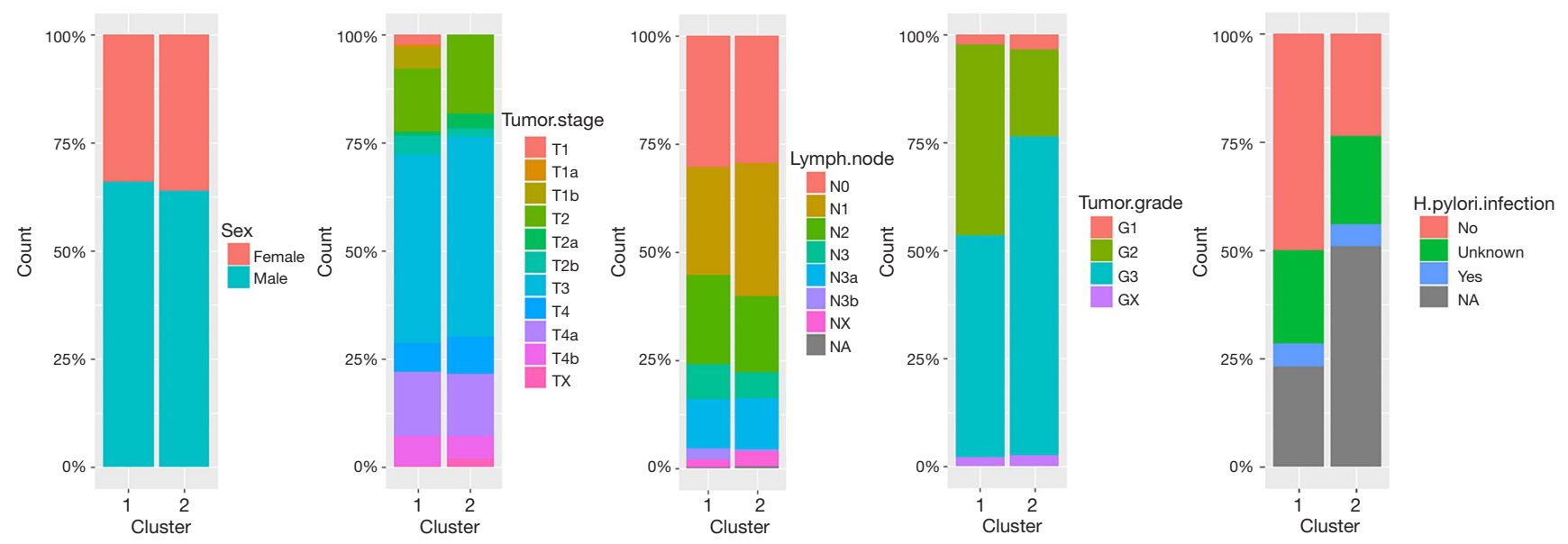

Figure 10 The CMTM-based stratification is an independent prognostic factor in GC patients and closely correlated with the STAD cohort mutation count. Percentage-staked bar plot showing sex distribution, tumor stage (T1/T1a/T1b/T2/T2a/T2b/T3/T4/T4a/T4b/ TX), metastatic lymph node status (N0/N1/N2/N3/N3a/N3b/NX/NA), differential tumor grade (G1/G2/G3/GX), and H. pylori status (No/Unknown/Yes/NA) in clusters \#1 and \#2. STAD, stomach adenocarcinoma. CMTM genes, chemokine-like factor-like MARVEL transmembrane domain-containing family genes.

In this research, CMTM1 was shown to be highly expressed in GC, which was significantly correlated with various clinical parameters, including cancer stage, lymph node metastasis status, and tumor grade. Also, a high expression level of CMTM1 was found to be closely correlated with poor PPS in GC patients in the survival analysis. These results suggest that CMTM1 has potential as a valuable molecular biomarker of GC, and its specific biological function and molecular mechanism in GC warrant attention in future.

The CMTM2 gene is located on chromosome 16q21 and is closely clustered with CMTM1, with which it shares a high level of amino acid sequence identity. In a study of 240 patients with diffuse-type GC, higher CMTM2 expression level was found to be associated with better OS (31). Guo et al. observed that CMTM2 was downregulated in HCC samples compared with paired adjacent normal tissues based on bioinformatics and immunohistochemistry (32). Their study also demonstrated the correlation between CMTM2 expression and HCC pathological grade. Further study provided verification that knockdown of CMTM2 in vitro was remarkably associated with downregulation of E-cadherin and $\beta$-catenin expression, and upregulation of $\mathrm{N}$-cadherin, Vimentin, ZEB1, and ZEB2. Also, CMTM2 was demonstrated to be involved in the inhibition of the invasion and migratory abilities of HCC cells (Huh-7 and SMMC7721), which was realized through the suppression of epithelial-mesenchymal transition (EMT) (33). Our results showed that elevated CMTM2 expression was closely related to favorable prognostic outcomes in patients with GC in terms of OS, PFS, and PPS. Based on the existing evidence, more indepth study and investigation of CMTM2 are warranted.

CMTM3 and CMTM4 genes are clustered on chromosome 16q22.1. Interestingly, the CMTM3 promoter differs from those of other CMTM family members due to its typical CpG island. CMTM3 is known to be involved in tumor suppression; however, the methylation of the CMTM3 promoter has been shown to suppress CMTM3 expression in various cancer types, such as gastric, male laryngeal, esophageal, breast, nasopharyngeal, and colon carcinomas (8,34-36). In the study of Li et al., CMTM3 was demonstrated to trigger G2 cell-cycle arrest and apoptosis in a p53-independent manner, leading to inhibition of the proliferative and migratory abilities of testicular cancer cells (NCCIT) (37). Moreover, by suppressing the epidermal growth factor (EGF)-mediated tumorigenicity via promoting Rab5 activity, and inhibiting GC metastasis via the STAT3/Twist1/EMT pathway, upregulation of CMTM3 was shown to be correlated with a better prognosis in GC patients (38-40). In order to find out whether CMTM3 participates in regulation of the tumor microenvironment of HCC, Zhao et al. investigated the expressions of chemokines and chemokine-associated genes 
of suppressor subsets derived from myeloid cells (41). However, the correlation between CMTM3 and the clinical parameters of GC patients remained unclear and warranted further study. In this study, CMTM3 was found to be upregulated in GC samples. In addition, CMTM3 expression was increased with advanced tumor stage, lymph node metastasis, high tumor grade, and $H$. pylori infection. The OS, PFS, and PPS were worse in GC patients with a high CMTM3 expression than in patients with a low expression. Together, our results suggest that CMTM3 may serve as a potential biomarker for GC and a guide for clinical treatment.

Among the CMTM genes, CMTM4 is more conserved than its fellow family members. Kittler et al. used an endoribonuclease-prepared short interfering RNA technique to demonstrate that CMTM4 was 1 of $37 \mathrm{hub}$ genes involved in the activation of cell division in HeLa cells (42). CMTM4 is known as a tumor suppressor, and its knockdown leads to cellular cleavage defects, resulting in binucleated cells following mitosis. The main underlying molecular mechanisms include the PI3K/AKT, ERK1/2, and STAT3 signaling pathways. In an important study by Mezzadra et al., CMTM4 was demonstrated to be a backup regulator of programmed cell death-ligand 1 (PD-L1) and was capable of inhibiting the ubiquitination of $\mathrm{PD}$ L1 protein (12). However, the difference in CMTM4 expression at both the protein and mRNA levels remains to be tested and awaits further research. The mutation status, methylation, microRNA silencing, and posttranscriptional regulation of CMTM4 expression in HCC have not been well studied to date and also await more in-depth answers. Similarly, there is still a great need for research dedicated to determining the prognostic value of CMTM4 in GC. One of the aims of our study was to elucidate the role of CMTM4 in GC. We found that higher levels of CMTM4 expression had an association with favorable OS, PFS, and PPS, indicating that CMTM4 could be a valuable biomarker for predicting GC prognosis.

As an evolutionarily conserved protein sharing $42 \%$ homology with CMTM3, CMTM5-v1 is the main alternative splicing form of CMTM5 in humans. The methylation status of the promoter in the CMTM5 gene mediates the activation of tumor-evading anticancer effects, which is similar to CMTM3 gene (9). CMTM5 has been discovered to be involved in a variety of cancers as a regulator of tumor suppression. For instance, a previous study showed that CMTM5-v1 has the ability to induce cancer cell apoptosis in various pancreatic cancer cells (AsPC-1,
BxPC-3, PANC-1, and MIA PaCa-2) via activating caspase 3,8 , and 9 (43). The same study also demonstrated that CMTM5-v1 has synergistic effects with TNF- $\alpha$. Conversely, our study showed that a high CMTM5 level in GC patients was predictive of poor OS, PFS, and PPS. Therefore, a longitudinal study is necessary for further investigation of the molecular mechanism of CMTM5 in GC.

CMTM6 is one of the CMTM family genes located on chromosome $3 \mathrm{p} 22.3$, which shares $55 \%$ homology with CMTM4. Expression of CMTM6 is widely found in normal tissues, primarily in the plasma membrane. At present, the mechanism controlling this linkage is elusive. However, in recent studies, CMTM6 was indicated to be a pivotal regulator of $\mathrm{PD}-\mathrm{L} 1$ expression and to be co-localized with PD-L1 $(12,13)$. With the help of the CRISPR-Cas9 technique, Burr et al. demonstrated that CMTM6 was capable of protecting internalized PD-L1 from lysosomal degradation. Furthermore, upregulation of CMTM6 could effectively recycle PD-L1 back to the cell surface (13). Additionally, Mezzadra et al. indicated that CMTM6 was able to stabilize PD-L1 via ubiquitination suppression, leading to T-cell inhibition (12). Another study suggested that overexpression of CMTM6 enhanced the therapeutic response to immune checkpoint inhibitors in advanced-stage non-small-cell lung cancer (44). Our study demonstrated that GC patients with high CMTM6 expression achieved better OS, PFS, and PPS than those with low CMTM6 expression, as evidenced by the KaplanMeier Plotter results. Since CMTM6 was highly expressed in cancer patients, it has the potential to be a prognostic marker in GC or to guide the administration of PD-1/PDL1 therapies.

The mRNA expression level of CMTM7 is extensively upregulated in healthy human tissues, and immune cells in particular. The CMTM7 protein is located in both the cytoplasm and cellular membrane. Some studies have suggested that CMTM7 is typically downregulated or absent in several cancer types, which could be ascribed to the abnormal methylation of $\mathrm{CpG}$ promoter and loss of heterozygosity. Through the induction of G1/S-phase arrest and suppressing the EGFR-PI3K/AKT pathway, upregulation of CMTM7 was able to inhibit cancer cell growth in KYSE410 and KYSE180 cancer cell lines (45). Some other studies suggested that the CMTM7 expression level could be adjusted dynamically with the help of 2 different transcription factors (FLI1 and SOX10) during tumor progression $(46,47)$. In another study, CMTM7 was found to be a suppressor in Rab5 activation as well as 
an activator of tumor growth and migration pathways in non-small cell lung cancer (48). In the current study, the CMTM7 expression level was found to be upregulated in GC and significantly associated with the clinical parameters of patients such as tumor stage, tumor grade, and lymph node metastasis status. Furthermore, we explored the correlation between survival of GC patients and the mRNA expression level of CMTM7. The results suggested that CMTM7 predicted poor PPS in patients with GC.

A previous study of Jin et al. showed that CMTM8 exhibited tumor-suppressive activity in various cell lines (HEK293, HeLa, and PC3) (49). The mechanism of these suppressive effects is attributable to the internalization of epidermal growth factor receptor (EGFR) being accelerated by CMTM8 regulation, which led to the attenuation of EGFR-mediated signaling pathway. In another study, CMTM8 was demonstrated to induce cancer cell apoptosis via either caspase-dependent or caspase-independent pathway (50). Similarly, upregulation of CMTM8 expression in bladder cancer was shown to suppress the growth, migratory, and invasive abilities of cancer cells in vivo and in vitro (14). In our study, CMTM8 mRNA expression was upregulated in GC, and we also observed that an elevated level of CMTM8 was related to a favorable survival time.

\section{Conclusions}

In the current study, we performed a comprehensive bioinformatics analysis to investigate the expression levels and prognostic value of CMTM family genes in GC, in an attempt to broaden our molecular understanding of GC, uncover novel therapeutic targets, and more accurately predict the prognosis of GC patients. Additionally, we performed an unsupervised hierarchical clustering analysis of a STAD cohort from TCGA. Two main subgroups were identified: the first with CMTM1/2/3/5/7 and the other with CMTM4/6/8. The first group exhibited shorter OS than the second group, suggesting that its signature was related to a poor prognosis. Thus, we suggest that a deeper knowledge of CMTM expression patterns could assist in diagnosing GC and improving individualized treatment strategies.

\section{Acknowledgments}

We would like to acknowledge the KEGG, cBioPortal, DAVID, Oncomine, GEPIA, STRING, TISIDB, TIMER,
TCGA, and UALCAN databases, which were used free of charge.

Funding: None.

\section{Footnote}

Reporting Checklist: The authors have completed the MDAR reporting checklist. Available at http://dx.doi.org/10.21037/ jgo-21-78

Conflicts of Interest: Both authors have completed the ICMJE uniform disclosure form (available at http://dx.doi. org/10.21037/jgo-21-78). The authors have no conflicts of interest to declare.

Ethical Statement: The authors are accountable for all aspects of the work in ensuring that questions related to the accuracy or integrity of any part of the work are appropriately investigated and resolved. This study was carried out with adherence to the stipulations of the Declaration of Helsinki (as revised in 2013). As the study did not include any experiments involving animal or human subjects performed by any of the authors, neither ethical approval nor patient consent were required.

Open Access Statement: This is an Open Access article distributed in accordance with the Creative Commons Attribution-NonCommercial-NoDerivs 4.0 International License (CC BY-NC-ND 4.0), which permits the noncommercial replication and distribution of the article with the strict proviso that no changes or edits are made and the original work is properly cited (including links to both the formal publication through the relevant DOI and the license). See: https://creativecommons.org/licenses/by-nc-nd/4.0/.

\section{References}

1. Han W, Lou Y, Tang J, et al. Molecular cloning and characterization of chemokine-like factor 1 (CKLF1), a novel human cytokine with unique structure and potential chemotactic activity. Biochem J 2001;357:127-35.

2. Duan HJ, Li XY, Liu C, et al. Chemokine-like factor-like MARVEL transmembrane domain-containing family in autoimmune diseases. Chin Med J (Engl) 2020;133:951-8.

3. Zhang JW, Liu TF, Chen XH, et al. Validation of aspirin response-related transcripts in patients with coronary artery disease and preliminary investigation on CMTM5 function. Gene 2017;624:56-65. 
4. Liu Q, Su Y, Jiang GC, et al. Change of CMTM7 expression, a potential tumor suppressor, is associated with poor clinical outcome in human non-small cell lung cancer. Chin Med J (Engl) 2013;126:3006-12.

5. $\mathrm{Hu} \mathrm{H}$, Chen JW, Xu KX, et al. Expressions of CMTM8 and E-cadherin in primary and metastatic clear cell renal cell carcinoma. Beijing Da Xue Xue Bao Yi Xue Ban 2013;45:537-41.

6. Li P, Liu K, Li L, et al. Reduced CMTM5 expression correlates with carcinogenesis in human epithelial ovarian cancer. Int J Gynecol Cancer 2011;21:1248-55.

7. Niu J, Li H, Zhang Y, et al. Aberrant expression of CKLFlike MARVEL transmembrane member 5 (CMTM5) by promoter methylation in myeloid leukemia. Leuk Res 2011;35:771-6.

8. Wang Y, Li J, Cui Y, et al. CMTM3, located at the critical tumor suppressor locus 16q22.1, is silenced by CpG methylation in carcinomas and inhibits tumor cell growth through inducing apoptosis. Cancer Res 2009;69:5194-201.

9. Shao L, Cui Y, Li H, et al. CMTM5 exhibits tumor suppressor activities and is frequently silenced by methylation in carcinoma cell lines. Clin Cancer Res 2007;13:5756-62.

10. Zhang W, Qi H, Mo X, et al. CMTM8 is frequently downregulated in multiple solid tumors. Appl Immunohistochem Mol Morphol 2017;25:122-8.

11. Xie J, Yuan Y, Liu Z, et al. CMTM3 is frequently reduced in clear cell renal cell carcinoma and exhibits tumor suppressor activities. Clin Transl Oncol 2014;16:402-9.

12. Mezzadra R, Sun C, Jae LT, et al. Identification of CMTM6 and CMTM4 as PD-L1 protein regulators. Nature 2017;549:106-10.

13. Burr ML, Sparbier CE, Chan YC, et al. CMTM6 maintains the expression of PD-L1 and regulates antitumour immunity. Nature 2017;549:101-5.

14. Gao D, Hu H, Wang Y, et al. CMTM8 inhibits the carcinogenesis and progression of bladder cancer. Oncol Rep 2015;34:2853-63.

15. Si J, Zhang P, Tian D, et al. CMTM1_v17 is associated with chemotherapy resistance and poor prognosis in nonsmall cell lung cancer. World J Surg Oncol 2017;15:34.

16. Zhang XY, Zhang PY. Gastric cancer: somatic genetics as a guide to therapy. J Med Genet 2017;54:305-12.

17. Franz JL, Cruz AB, Jr. The treatment of gastric cancer with combined surgical resection and chemotherapy. J Surg Oncol 1977;9:131-7.

18. Coburn N, Cosby R, Klein L, et al. Staging and surgical approaches in gastric cancer: A systematic review. Cancer Treat Rev 2018;63:104-15.

19. Tang Z, Kang B, Li C, et al. GEPIA2: an enhanced web server for large-scale expression profiling and interactive analysis. Nucleic Acids Res 2019;47:W556-60.

20. Ritchie ME, Phipson B, Wu D, et al. limma powers differential expression analyses for RNA-sequencing and microarray studies. Nucleic Acids Res 2015;43:e47.

21. Uhlen $M$, Zhang C, Lee S, et al. A pathology atlas of the human cancer transcriptome. Science 2017;357:ean2507.

22. Cerami E, Gao J, Dogrusoz U, et al. The cBio cancer genomics portal: an open platform for exploring multidimensional cancer genomics data. Cancer Discov 2012;2:401-4.

23. Gao J, Aksoy BA, Dogrusoz U, et al. Integrative analysis of complex cancer genomics and clinical profiles using the cBioPortal. Sci Signal 2013;6:pl1.

24. Hoadley KA, Yau C, Hinoue T, et al. Cell-of-origin patterns dominate the molecular classification of 10,000 tumors from 33 types of cancer. Cell 2018;173:291-304.e6.

25. Huang da W, Sherman BT, Lempicki RA. Systematic and integrative analysis of large gene lists using DAVID bioinformatics resources. Nat Protoc 2009;4:44-57.

26. Huang da W, Sherman BT, Lempicki RA. Bioinformatics enrichment tools: paths toward the comprehensive functional analysis of large gene lists. Nucleic Acids Res 2009;37:1-13.

27. Han W, Ding P, Xu M, et al. Identification of eight genes encoding chemokine-like factor superfamily members 1-8 (CKLFSF1-8) by in silico cloning and experimental validation. Genomics 2003;81:609-17.

28. Sánchez-Pulido L, Martín-Belmonte F, Valencia A, et al. MARVEL: a conserved domain involved in membrane apposition events. Trends Biochem Sci 2002;27:599-601.

29. Wu K, Li X, Gu H, et al. Research advances in CKLF-like MARVEL transmembrane domain-containing family in non-small cell lung cancer. Int J Biol Sci 2019;15:2576-83.

30. Wang J, Zhang G, Zhang Y, et al. CMTM1_v17 is a novel potential therapeutic target in breast cancer. Oncol Rep 2014;32:1829-36.

31. Choi JH, Kim YB, Ahn JM, et al. Identification of genomic aberrations associated with lymph node metastasis in diffuse-type gastric cancer. Exp Mol Med 2018;50:1-11.

32. Guo X, Zhang S, Tan S, et al. Downregulated CMTM2 poses potential clinical significance in hepatocellular carcinoma. DNA Cell Biol 2020;39:683-9.

33. Zhang S, Tian R, Bei C, et al. Down-regulated CMTM2 promotes epithelial-mesenchymal transition 
in hepatocellular carcinoma. Onco Targets Ther 2020;13:5731-41.

34. Shen Z, Chen X, Li Q, et al. Elevated methylation of CMTM3 promoter in the male laryngeal squamous cell carcinoma patients. Clin Biochem 2016;49:1278-82.

35. Han T, Shu T, Dong S, et al. Chemokine-like factor-like MARVEL transmembrane domain-containing 3 expression is associated with a favorable prognosis in esophageal squamous cell carcinoma. Oncol Lett 2017;13:2982-8.

36. Li J, Chen C, Bi X, et al. DNA methylation of CMTM3, SSTR2, and MDFI genes in colorectal cancer. Gene 2017;630:1-7.

37. Li Z, Xie J, Wu J, et al. CMTM3 inhibits human testicular cancer cell growth through inducing cell-cycle arrest and apoptosis. PLoS One 2014;9:e88965.

38. Su Y, Lin Y, Zhang L, et al. CMTM3 inhibits cell migration and invasion and correlates with favorable prognosis in gastric cancer. Cancer Sci 2014;105:26-34.

39. Yuan W, Li T, Mo X, et al. Knockdown of CMTM3 promotes metastasis of gastric cancer via the STAT3/ Twist1/EMT signaling pathway. Oncotarget 2016;7:29507-19.

40. Yuan W, Liu B, Wang X, et al. CMTM3 decreases EGFR expression and EGF-mediated tumorigenicity by promoting Rab5 activity in gastric cancer. Cancer Lett 2017;386:77-86.

41. Zhao $\mathrm{W}, \mathrm{Xu} \mathrm{Y}, \mathrm{Xu}$ J, et al. Subsets of myeloid-derived suppressor cells in hepatocellular carcinoma express chemokines and chemokine receptors differentially. Int Immunopharmacol 2015;26:314-21.

42. Kittler R, Putz G, Pelletier L, et al. An endoribonucleaseprepared siRNA screen in human cells identifies genes essential for cell division. Nature 2004;432:1036-40.

Cite this article as: Liang Z, Xie J, Huang L, Huang Y, Zhang Y, Ma R, Zheng Z, Wang Q, Li X. Comprehensive analysis of the prognostic value of the chemokine-like factor-like MARVEL transmembrane domain-containing family in gastric cancer. J Gastrointest Oncol 2021;12(2):388-406. doi: 10.21037/ jgo-21-78
43. Guo X, Li T, Wang Y, et al. CMTM5 induces apoptosis of pancreatic cancer cells and has synergistic effects with TNF-alpha. Biochem Biophys Res Commun 2009;387:139-42.

44. Zugazagoitia J, Liu Y, Toki M, et al. Quantitative assessment of CMTM6 in the tumor microenvironment and association with response to PD-1 pathway blockade in advanced-stage non-small cell lung cancer. J Thorac Oncol 2019;14:2084-96.

45. Li H, Li J, Su Y, et al. A novel 3p22.3 gene CMTM7 represses oncogenic EGFR signaling and inhibits cancer cell growth. Oncogene 2014;33:3109-18.

46. Miao B, Bauer AS, Hufnagel K, et al. The transcription factor FLI1 promotes cancer progression by affecting cell cycle regulation. Int J Cancer 2020;147:189-201.

47. Jin Y, Qin X, Jia G. SOX10-dependent CMTM7 expression inhibits cell proliferation and tumor growth in gastric carcinoma. Biochem Biophys Res Commun 2018;507:91-9.

48. Liu B, Su Y, Li T, et al. CMTM7 knockdown increases tumorigenicity of human non-small cell lung cancer cells and EGFR-AKT signaling by reducing Rab5 activation. Oncotarget 2015;6:41092-107.

49. Jin C, Ding P, Wang Y, et al. Regulation of EGF receptor signaling by the MARVEL domain-containing protein CKLFSF8. FEBS Lett 2005;579:6375-82.

50. Jin C, Wang Y, Han W, et al. CMTM8 induces caspasedependent and -independent apoptosis through a mitochondria-mediated pathway. J Cell Physiol 2007;211:112-20.

(English Language Editor: J. Reynolds) 